\title{
Mathematical Modeling in Shell Structure Analysis Tasks
}

\author{
Alexey Semenov \\ Department of Information Technologies, Saint Petersburg State University of Architecture and Civil Engineering, Saint \\ Petersburg, RUSSIAN FEDERATION \\ e-mail: sw.semenov@gmail.com
}

\section{SUMMARY}

Studying shell structures while accounting for all necessary factors is a significantly nonlinear problem that requires serious mathematical tools and sufficient computing capacities. We propose an approach to solving such problems based on the following numerical methods. We propose the use of the Ritz method, the best parameter continuation method, and the Euler method under static loading. Under dynamic loading, the Kantorovich and the Rosenbrock methods are used. Software implementation was carried out using the Maple analytical software package. This paper also provides examples of simulating the deformation process in shell structures.

KEY WORDS: computer simulation; shell structures; buckling; Ritz method; method of L.V. Kantorovich.

\section{INTRODUCTION}

Improved computing tools have given rise to a new method of investigating scientific problems: the method of computational experiment. Currently, it is the predominant way to study complex processes and phenomena. Such complex processes include deformation in thin-walled structures, especially shells. In such structures, buckling with bulges and dents is observed under various loads $[2,10,18]$.

In [2], the theoretical foundations, methods, and algorithms developed to analyze the stability and postbuckling behavior of thin elastic axisymmetric shells are discussed. The procedure of determining an isolated solution, whose existence was predicted by V. I. Feodosiev, is considered within the framework of studying the process of nonlinear deformation of a corrugated membrane loaded by external pressure.

Maksimyuk, Storozhuk, and Chernyshenko [10] discusses variational finite-difference methods of solving linear and nonlinear problems for thin and non-thin shells (plates) made of homogeneous isotropic (metallic) and orthotropic (composite) materials are analyzed and their classification principles and structure. The stress-strain state of metallic and composite shells of complex geometry is analyzed numerically. 
Studying shell structures while accounting for all necessary factors is a significantly nonlinear problem that requires serious mathematical tools and sufficient computing capacities $[1,3,5$, $7,17]$.

Fan et al. [1] present an analytical study on the critical dynamic buckling load of cylindrical shells with arbitrary axisymmetric thickness variation under uniform external pressure that is a function of time. Based on the Donnell simplified principle, the equilibrium and compatibility equations of cylindrical shells were transformed into ordinary differential equations in nondimensional form. The buckling capacity of cylindrical shells under dynamic external pressure was discussed considering the effects of loading speed and thickness variation parameters.

Kumar [7] presents a comprehensive literature review on the application of the Rayleigh-Ritz method to analyze vibration, static and buckling characteristics of beams, shells, and plates using different theories.

Proposes an approach to solving such problems based on the following numerical methods:

- for strength and buckling problems under static loading, the Ritz method $[9,13,15,16,19$, 20]; the best parameter continuation method [8]; and the Euler method for solving the initial problem;

- for strength and buckling problems, as well as problems of nonlinear oscillations under dynamic loading, the Kantorovich method [11] and the Rosenbrock method [14].

\section{MATHEMATICAL MODEL}

Let us examine thin-walled shells under external mechanical loading. The geometry of these structures is defined by the Lamé coefficients $A, B$ and radii of principal curvatures $R_{1}, R_{2}$ along the $x, y$ coordinates, respectively. The Lamé coefficients are used to transform vectors when moving from one coordinate system to another. In this case, from the global Cartesian coordinate system $(X, Y, Z)$ to the local curvilinear coordinate system $(x, y, z)$ :

$$
A=\sqrt{\left(\frac{\partial X}{\partial x}\right)^{2}+\left(\frac{\partial Y}{\partial x}\right)^{2}+\left(\frac{\partial Z}{\partial x}\right)^{2}}, B=\sqrt{\left(\frac{\partial X}{\partial y}\right)^{2}+\left(\frac{\partial Y}{\partial y}\right)^{2}+\left(\frac{\partial Z}{\partial y}\right)^{2}}
$$

Let us use a mathematical model of the Timoshenko (Mindlin-Reissner) type, which takes into account transverse shears, material orthotropy, and geometric nonlinearity [12]. According to this model, in the case of static problems, three functions characterizing displacement of the coordinate surface points $U(x, y), V(x, y), W(x, y)$ and two functions characterizing the normal rotation angles in the planes $x O z, y O z,\left(\Psi_{x}(x, y), \Psi_{y}(x, y)\right)$ will be the unknown functions. In the case of dynamic problems, $U(x, y, t), V(x, y, t), W(x, y, t), \Psi_{x}(x, y, t), \Psi_{y}(x, y, t)$ will be the unknown functions. Taking into account geometric nonlinearity, we can analyze not only the stress-strain state of a shell but also its stability.

The following assumptions will be valid:

The stresses arising in the shell in the direction perpendicular to the median surface are considered negligible compared to other stresses $\left(\sigma_{z}=0\right)$.

1. For thin shells $\frac{h}{\min \left\{R_{1} ; R_{2}\right\}}<\frac{1}{20}$. Here $h$ - shell thickness. 
2. When deriving the governing equations of the theory of shells, quantities of a larger order of smallness are neglected than $\frac{h}{\min \left\{R_{1} ; R_{2}\right\}}$.

3. The hypothesis holds that the rectilinear element of the shell, initially normal to the middle surface, remains rectilinear even in the process of deformation, but not necessarily normal.

4. All components of the strain tensor at any point of the shell are small compared to unity $\varepsilon_{i j}<<1, i, j=1,2,3$. The squared angles of rotation of the normal to the middle surface of the shell during its bending have the same order of smallness $\Psi_{i}^{2}<<1, i, j=1,2$. Under these conditions, the normal displacements of the middle surface of the shell can be of the order of thickness or even more but are small in comparison with other dimensions. Such bending of the shell is called average and is described by nonlinear dependences.

5. For shallow shells, the ratio of the shell lifting boom $d$ to the smallest linear dimension in the plan is less than $1 / 5$.

\subsection{STATIC PROBLEMS}

This model is based on the functional of total potential deformation energy, which can be written in the following form:

$$
\begin{gathered}
E_{s}=\frac{1}{2} \int_{a_{1}}^{a} \int_{0}^{b}\left(N_{x} \varepsilon_{x}+N_{y} \varepsilon_{y}+\frac{1}{2}\left(N_{x y}+N_{y x}\right) \gamma_{x y}+M_{x} \chi_{1}+M_{y} \chi_{2}+\right. \\
\left.+\left(M_{x y}+M_{y x}\right) \chi_{12}+Q_{x}\left(\Psi_{x}-\theta_{1}\right)+Q_{y}\left(\Psi_{y}-\theta_{2}\right)-2\left(q W+P_{x} U+P_{y} V\right)\right) A B d x d y,
\end{gathered}
$$

where $q, P_{x}, P_{y}$ denote load components; $a_{1} \leq x \leq a, 0 \leq y \leq b$ - shell area boundaries; $N_{x}, N_{y}$ denote normal forces in the direction of the $x, y$ coordinates; $N_{x y}, N_{y x}$ denote shear forces in the corresponding plane $x O y ; M_{x}, M_{y}$ denote bending moments; $M_{x y}, M_{y x}$ denote torque moments; $Q_{x}, Q_{y}$ denote transverse forces in the planes $x O z$ and $y O z$, which are defined by the following relationships:

$$
\begin{gathered}
N_{x}=\frac{E_{1} h}{1-\mu_{12} \mu_{21}}\left(\varepsilon_{x}+\mu_{21} \varepsilon_{y}\right), \quad N_{y}=\frac{E_{2} h}{1-\mu_{12} \mu_{21}}\left(\varepsilon_{y}+\mu_{12} \varepsilon_{x}\right), \quad N_{x y}=N_{y x}=G_{12} h \gamma_{x y}, \\
M_{x}=\frac{E_{1} h^{3}}{12\left(1-\mu_{12} \mu_{21}\right)}\left(\chi_{1}+\mu_{21} \chi_{2}\right), \quad M_{y}=\frac{E_{2} h^{3}}{12\left(1-\mu_{12} \mu_{21}\right)}\left(\chi_{2}+\mu_{12} \chi_{1}\right), \\
M_{x y}=M_{y x}=\frac{G_{12} h^{3}}{6} \chi_{12}, \quad Q_{x}=G_{13} k h\left(\Psi_{x}-\theta_{1}\right), \quad Q_{y}=G_{23} k h\left(\Psi_{y}-\theta_{2}\right) .
\end{gathered}
$$

Here $E_{1}, E_{2}$ denote elasticity moduli in the directions $x, y ; h$ - shell thickness; $k=5 / 6$; $G_{12}, G_{13}, G_{23}$ denote shear moduli in the planes $x O y, x O z, y O z$, respectively; $\mu_{12}, \mu_{21}$ denote Poisson's ratios; $\varepsilon_{x}, \varepsilon_{y}$ denotes tensile strains; $\gamma_{x y}$ denotes shear strains in the plane $x O y$; $\chi_{1}, \chi_{2}, \chi_{12}$ denote functions of change in curvature and torsion as follows: 


$$
\begin{gathered}
\mathcal{E}_{x}=\frac{1}{A} \frac{\partial U}{\partial x}+\frac{1}{A B} V \frac{\partial A}{\partial y}-k_{x} W+\frac{1}{2} \theta_{1}^{2}, \quad \varepsilon_{y}=\frac{1}{B} \frac{\partial V}{\partial y}+\frac{1}{A B} U \frac{\partial B}{\partial x}-k_{y} W+\frac{1}{2} \theta_{2}^{2}, \\
\gamma_{x y}=\frac{1}{A} \frac{\partial V}{\partial x}+\frac{1}{B} \frac{\partial U}{\partial y}-\frac{1}{A B} U \frac{\partial A}{\partial y}-\frac{1}{A B} V \frac{\partial B}{\partial x}+\theta_{1} \theta_{2}, \\
\theta_{1}=-\left(\frac{1}{A} \frac{\partial W}{\partial x}+k_{x} U\right), \quad \theta_{2}=-\left(\frac{1}{B} \frac{\partial W}{\partial y}+k_{y} V\right), \quad k_{x}=\frac{1}{R_{1}}, \quad k_{y}=\frac{1}{R_{2}}, \\
\chi_{1}=\frac{1}{A} \frac{\partial \Psi_{x}}{\partial x}+\frac{1}{A B} \frac{\partial A}{\partial y} \Psi_{y}, \quad \chi_{2}=\frac{1}{B} \frac{\partial \Psi_{y}}{\partial y}+\frac{1}{A B} \frac{\partial B}{\partial x} \Psi_{x}, \\
\chi_{12}=\frac{1}{2}\left(\frac{1}{A} \frac{\partial \Psi_{y}}{\partial x}+\frac{1}{B} \frac{\partial \Psi_{x}}{\partial y}-\frac{1}{A B} \frac{\partial B}{\partial x} \Psi_{y}-\frac{1}{A B} \frac{\partial A}{\partial y} \Psi_{x}\right) .
\end{gathered}
$$

Due to the geometry of the structures under consideration, large displacements occur along the vertical axis, which leads to the nonlinearity of the deformation process. Compared to deformation along the vertical axis, transverse shears are significantly less, however, they still matter, especially for stiffened structures.

The form of the transverse load is specified as follows:

$$
q=q_{0}\left(a_{11}+a_{21} x+a_{31} x^{2}\right)\left(a_{12}+a_{22} y+a_{32} y^{2}\right)+q_{s v}
$$

where $q_{0}$ denotes the value of the transverse load, $M P a ; q_{S V}$ denotes the own weight of the shell, $M P a ; a_{i j}$ - coefficients that allow setting the shape of the distribution of the applied load. For example, for a linearly increasing load, it will be $a_{11}=-a_{1} /\left(a-a_{1}\right), a_{21}=1 /\left(a-a_{1}\right), a_{31}=0$, $a_{12}=0, a_{22}=1 / b, a_{32}=0$ and for an evenly distributed load, it will be $a_{11}=1, a_{21}=0, a_{31}=0$, $a_{12}=1, a_{22}=0, a_{32}=0$.

\subsection{DYNAMIC PROBLEMS}

Modeling of dynamic problems implies that not only the potential but also the kinetic deformation energy is considered. When considered together, the total deformation energy of a structure can be written using the following functional:

$$
I=\int_{t_{0}}^{t_{1}}\left(E_{k}-E_{s}\right) d t
$$

where $t$ denotes time; $E_{k}$ denotes kinetic energy of system deformation as follows:

$$
E_{k}=\frac{\rho}{2} \int_{a_{1}}^{a} \int_{0}^{b}\left\{h\left[\left(\frac{\partial U}{\partial t}\right)^{2}+\left(\frac{\partial V}{\partial t}\right)^{2}+\left(\frac{\partial W}{\partial t}\right)^{2}\right]+\frac{h^{3}}{12}\left[\left(\frac{\partial \Psi_{x}}{\partial t}\right)^{2}+\left(\frac{\partial \Psi_{y}}{\partial t}\right)^{2}\right]\right\} A B d x d y
$$

Here $\rho$ denotes shell material density.

\subsection{DIMENSIONLESS PARAMETERS}

To ensure adequate application of the numerical methods, we introduce the following dimensionless parameters: 


$$
\begin{gathered}
\xi=\frac{x}{a}, \quad \eta=\frac{y}{b}, \quad \bar{\lambda}=\frac{a A}{b B}, \quad k_{\xi}=h k_{x}, \quad k_{\eta}=h k_{y}, \quad \bar{A}=\frac{a A}{h}, \quad \bar{B}=\frac{b B}{h}, \\
\bar{U}=\frac{a U A}{h^{2}}, \quad \bar{V}=\frac{b V B}{h^{2}}, \quad \bar{W}=\frac{W}{h}, \quad \bar{\Psi}_{x}=\frac{\Psi_{x} a A}{h}, \quad \bar{\Psi}_{y}=\frac{\Psi_{y} b B}{h}, \\
\bar{P}=\frac{a^{4} A^{4} q}{h^{4} E_{1}}, \quad \bar{t}=\frac{h}{a^{2} A^{2}} \sqrt{\frac{E_{1}}{\left(1-\mu_{12} \mu_{21}\right) \rho}} \cdot t .
\end{gathered}
$$

Thus, we pass to a new coordinate system $(\xi, \eta, \bar{z})$, and $\bar{U}(\xi, \eta), \bar{V}(\xi, \eta), \bar{W}(\xi, \eta), \bar{\Psi}_{x}(\xi, \eta), \bar{\Psi}_{y}(\xi, \eta)$ are now the unknown functions. The conversion to dimensionless parameters was described in detail in [6].

\section{ALGORITHM FOR THE SOLUTION OF STRENGTH AND BUCKLING PROBLEMS}

\subsection{STATIC PROBLEMS}

To solve a buckling analysis problem in a shell structure, we need to find the minimum of the functional (1). For that purpose, let us apply the Ritz method to reduce the variational problem of finding the functional minimum to solve a system of nonlinear algebraic equations [4]. In this case, the unknown dimensionless functions can be presented in the following form:

$$
\begin{gathered}
\bar{U}=\sum_{k=1}^{n} \sum_{l=1}^{n} U_{k l} X_{1}^{k} Y_{1}^{l}, \quad \bar{V}=\sum_{k=1 l=1}^{n} \sum_{k l}^{n} V_{k} X_{2}^{k} Y_{2}^{l}, \quad \bar{W}=\sum_{k=1 l=1}^{n} \sum_{k l}^{n} W_{k} X_{3}^{k} Y_{3}^{l}, \\
\bar{\Psi}_{X}=\sum_{k=1}^{n} \sum_{l=1}^{n} \Psi_{X_{k l}} X_{4}^{k} Y_{4}^{l}, \quad \bar{\Psi}_{y}=\sum_{k=1 l=1}^{n} \sum^{n} \Psi_{y_{k l}} X_{5}^{k} Y_{5}^{l},
\end{gathered}
$$

where $U_{k l}, V_{k l}, W_{k l}, \Psi_{x_{k l}}, \Psi_{y_{k l}}$ denote unknown numerical parameters, $X_{1}^{k}, \ldots, X_{5}^{k}$ and $Y_{1}^{l}, \ldots, Y_{5}^{l}$ denote known approximating functions with arguments $\xi$ and $\eta$, satisfying the given boundary conditions. The boundary conditions for a particular structure are determined based on the type of shell contour support.

Let us consider thin-walled shells with pin support along the contour (at $x=0, x=a: U=V=W=M_{x}=\Psi_{y}=0 ;$ at $\left.y=0, y=b: U=V=W=M_{y}=\Psi_{x}=0\right)$. Then, the following trigonometric functions can be considered as approximating ones:

$$
\begin{gathered}
X_{1}^{k}=\sin (2 k \pi \xi), \quad X_{2}^{k}=\sin ((2 k-1) \pi \xi), \quad X_{3}^{k}=\sin ((2 k-1) \pi \xi), \\
X_{4}^{k}=\cos ((2 k-1) \pi \xi), \quad X_{5}^{k}=\sin ((2 k-1) \pi \xi), \\
Y_{1}^{l}=\sin ((2 l-1) \pi \eta), \quad Y_{2}^{l}=\sin (2 l \pi \eta), \quad Y_{3}^{l}=\sin ((2 l-1) \pi \eta), \\
Y_{4}^{l}=\sin ((2 l-1) \pi \eta), \quad Y_{5}^{l}=\cos ((2 l-1) \pi \eta) .
\end{gathered}
$$

In the case of clamped fixing of the contour, it is necessary to use: 


$$
\begin{gathered}
X_{1}^{k}=\sin (2 k \pi \xi), \quad X_{2}^{k}=\sin ((2 k-1) \pi \xi), \quad X_{3}^{k}=\sin ((2 k-1) \pi \xi), \\
X_{4}^{k}=\sin ((2 k-1) \pi \xi), \quad X_{5}^{k}=\sin ((2 k-1) \pi \xi), \\
Y_{1}^{l}=\sin ((2 l-1) \pi \eta), \quad Y_{2}^{l}=\sin (2 l \pi \eta), \quad Y_{3}^{l}=\sin ((2 l-1) \pi \eta), \\
Y_{4}^{l}=\sin ((2 l-1) \pi \eta), \quad Y_{5}^{l}=\sin ((2 l-1) \pi \eta) .
\end{gathered}
$$

By substituting Eq. (9) to Eq. (1), we move from the functional $E_{s}$ to the function $E_{s f}$. To find the minimum, we need to find derivatives of the function $E_{s f}$ with respect to all unknown numerical parameters and then equate them to zero:

$$
\frac{\partial E_{s f}}{\partial U_{k l}}=0, \frac{\partial E_{s f}}{\partial V_{k l}}=0, \frac{\partial E_{s f}}{\partial W_{k l}}=0, \frac{\partial E_{s f}}{\partial \Psi_{x_{k l}}}=0, \frac{\partial E_{s f}}{\partial \Psi_{y_{k l}}}=0, \quad k, l=\overline{1, n} .
$$

As a result, we obtain a system of nonlinear algebraic equations. To solve them, we use the best parameter continuation method, which makes it possible to reduce the solution of a nonlinear system to the solution of the initial problem for a system of ordinary differential equations. It is proposed to take the length of the arc of the solution set curve $\lambda$ as the best parameter.

When solving nonlinear systems of equations, various unknown variables, that are present in the system of equations, can be selected as parameters for the continuation of the solution. However, selecting parameters, such as displacement coefficients or the load parameter leads to significant computational difficulties. In the $[8,21,22]$, it is proposed to choose a universal ("best") parameter, that will avoid computational difficulties. This is the arc length parameter $\lambda$.

The load parameter $\bar{P}$ is equally weighted with other unknown variables, therefore, we need to solve the problem in dimensionless form. In the case of adaptive grid selection, the best parameter continuation method makes it possible to find upper and lower critical loads, as well as bifurcation points, because the load parameter may decrease when bypassing special points.

Let us denote the following:

$$
X=\left(U_{k l}, V_{k l}, W_{k l}, \Psi_{x_{k l}}, \Psi_{y_{k l}}\right)^{T}, \quad \tilde{X}=\left(U_{k l}, V_{k l}, W_{k l}, \Psi_{x_{k l}}, \Psi_{y_{k l}}, \bar{P}\right)^{T}, \quad k, l=\overline{1, n},
$$

and denote the system of nonlinear equations $(10)$ as $F(\tilde{X})=0$.

It is known that when shells are not subjected to any load, displacements and normal rotation angles are equal to zero. Therefore, the initial value of $\tilde{X}$ is defined by zero vector of dimension $5 n^{2}+1$ :

$$
\tilde{X}^{0}=(0, \ldots, 0)^{T} .
$$

It should be noted that the length of the arc $\lambda$ is not included in the system of equations in explicit form. The parameter has the following relationship with the variables:

$$
(d \lambda)^{2}=\sum_{k=1}^{n} \sum_{l=1}^{n}\left[\left(d U_{k l}\right)^{2}+\left(d V_{k l}\right)^{2}+\left(d W_{k l}\right)^{2}+\left(d \Psi_{x_{k l}}\right)^{2}+\left(d \Psi_{y_{k l}}\right)^{2}\right]+(d \bar{P})^{2} .
$$

By differentiating the system of equations (10) with respect to $\lambda$ and assuming that all components of the vector $\tilde{X}$ depend on it, we get a system of $5 n$ ordinary differential equations: 


$$
\bar{J} \cdot \frac{d \tilde{X}}{d \lambda}=\left[\begin{array}{c}
0 \\
\vdots \\
0
\end{array}\right]
$$

with the initial conditions (12) at $\lambda_{0}=0$.

Here $\bar{J}=\partial F(\tilde{X}) / \partial \tilde{X}$ denotes augmented Jacobi matrix (with $5 n^{2}$ rows and $5 n^{2}+1$ columns), and $J=\partial F(X) / \partial X$ denotes ordinary Jacobi matrix of vector function $F$ (needed to calculate the determinant at each step of solution continuation, which makes it possible to detect special points based on criterion $\operatorname{det}(J)=0$ ).

By supplementing the system of equations (14) with the relationship (13), we obtain a closed system of the $5 n+1$ st ordinary differential equation:

$$
\left[\begin{array}{c}
\bar{J} \\
\left(\frac{d \tilde{X}}{d \lambda}\right)^{T}
\end{array}\right] \cdot \frac{d \tilde{X}}{d \lambda}=\left[\begin{array}{c}
0 \\
\vdots \\
0 \\
1
\end{array}\right],
$$

which the process of best parameter continuation for the system of nonlinear equations (10) reduces to at each step.

The system (15) is nonlinear relative to $d \tilde{X} / d \lambda$. To solve it, we use the option of the step-bystep continuation process suggested in the monograph [21]. The authors of the monograph suggest substituting the last row $(d \tilde{X} / d \lambda)^{T}$ in the matrix of the system (15) with vector $\left(d \tilde{X}^{*} / d \lambda\right)^{T}$, which at each step is considered equal to the vector calculated in the previous step. We can then proceed with the explicit scheme of the Euler method to solve the initial problem (15), (12) in the form of a system of linear algebraic equations with respect to $(d \tilde{X} / d \lambda)^{T}[22]$.

Thus, the solution of the nonlinear system of algebraic equations reduces to the successive solution of the systems of linear algebraic equations with respect to the increments of the required functions $\Delta \tilde{X}$. The greater the difference between the vectors $d \tilde{X} / d \lambda$ at the current and previous steps, the worse the system matrix conditioning, resulting in a less stable computing process. In this regard, it is reasonable to ensure adaptive grid selection to increase the accuracy of calculations.

The buckling load is detected as follows: the load/deflection diagram is analyzed, where a small change in the load corresponds to a significant change in the deflection (Lyapunov criterion), and snap-through buckling (transition to a new equilibrium state) occurs. The moment when the determinant of the Jacobi matrix $J$ becomes zero also corresponds to the critical load.

It should be noted that condition $\operatorname{det}=0$ may also correspond to lower critical loads and bifurcation points.

The search for the maximum allowable load that, once exceeded, results in strength loss, where damage to the material begins (or plastic deformations occur) at some point in the 
structure, will be performed at each step when continuing the solution by analyzing the values of stress components (criterion of maximum stresses):

$$
F_{1}^{-} \leq \sigma_{x} \leq F_{1}^{+}, \quad F_{2}^{-} \leq \sigma_{y} \leq F_{2}^{+}, \quad\left|\tau_{x y}\right| \leq F_{12} .
$$

Here $F_{1}^{+}, F_{2}^{+}$- ultimate tensile strength in the directions of orthotropy $1,2[\mathrm{MPa}] ; \mathrm{F}_{1}^{-}, \mathrm{F}_{2}^{-}-$ ultimate compressive strength in the directions of orthotropy $1,2[\mathrm{MPa}] ; F_{12}$ - ultimate shear strength in the plane of orthotropy $[\mathrm{MPa}]$.

In this case, the Maple analytical software package is the best option for software implementation since fairly intensive symbolic computations are required.

Verification of the proposed technique for the static problem (comparison with the results obtained by other authors) was shown in [16].

\subsection{DYNAMIC PROBLEMS}

The calculation algorithm is based on the Kantorovich method (for reducing the system of differential equations for functions of three variables to a system of ordinary differential equations (ODE) with respect to functions of only one variable $t$ ) and the Rosenbrock method (for the numerical solution of rigid ODE systems).

The Kantorovich method is used to reduce the multi-dimensional functional to a onedimensional functional. For that purpose, the required displacement functions and functions of the normal rotation angles are presented in the following form:

$$
\begin{gathered}
\bar{U}=\sum_{k=1}^{n} \sum_{l=1}^{n} U_{k l}(\bar{t}) X_{1}^{k} Y_{1}^{l}, \quad \bar{V}=\sum_{k=1}^{n} \sum_{l=1}^{n} V_{k l}(\bar{t}) X_{2}^{k} Y_{2}^{l}, \quad \bar{W}=\sum_{k=1 l=1}^{n} \sum^{n} W_{k l}(\bar{t}) X_{3}^{k} Y_{3}^{l}, \\
\bar{\Psi}_{X}=\sum_{k=1}^{n} \sum_{l=1}^{n} \Psi_{X_{k l}}(\bar{t}) X_{4}^{k} Y_{4}^{l}, \quad \bar{\Psi}{ }_{y}=\sum_{k=1}^{n} \sum_{l=1}^{n} \Psi_{y_{k l}}(\bar{t}) X_{5}^{k} Y_{5}^{l},
\end{gathered}
$$

where $U_{k l}-\Psi_{y k l}$ denotes unknown functions of the variable $\bar{t} ; X_{1}^{k}, \ldots, X_{5}^{k}$ and $Y_{1}^{l}, \ldots, Y_{5}^{l}$ denote known approximating functions with arguments $\xi$ and $\eta$, satisfying the given boundary conditions.

The functions (17) are then substituted into the functional of the total deformation energy of the shell (5). After calculating the integrals of the known functions over variables $\xi$ and $\eta$, the functional $\bar{I}$ becomes a one-dimensional functional of the functions $U_{k l}(\bar{t})-\Psi_{y k l}(\bar{t})$. Equations of motion, which are an ODE system, are obtained based on the conditions for this functional minimum $\delta \bar{I}=0$. The system thus derived is called a multi-dimensional variant of the Euler-Lagrange equation:

$$
\frac{d}{d \bar{t}} \frac{\partial\left(\bar{E}_{k}-\bar{E}_{s}\right)}{\partial \bar{X}_{j}(\bar{t})}-\frac{\partial\left(\bar{E}_{k}-\bar{E}_{s}\right)}{\partial \bar{X}_{j}(\bar{t})}=0, \quad j=1,2, \ldots, 5 n^{2},
$$

where $\bar{X}(\bar{t})=\left(U_{k l}(\bar{t}), V_{k l}(\bar{t}), W_{k l}(\bar{t}), \Psi_{x k l}(\bar{t}), \Psi_{y k l}(\bar{t})\right)^{T}, \quad k, l=\overline{1, n}$, and the dot denote the time derivative. 
Since the derivatives of the required functions with respect to the variable $\bar{t}$ are contained only in the expression for the kinetic energy, and the functions themselves only in the expression for $\bar{E}_{s}$, then the following is true:

$$
\frac{d}{d \bar{t}} \frac{\partial \bar{E}_{k}}{\partial \bar{X}_{j}(\bar{t})}+\frac{\partial \bar{E}_{s}}{\partial \bar{X}_{j}(\bar{t})}=0, j=1,2, \ldots, 5 n^{2} .
$$

Moreover, $\frac{\partial \bar{E}_{s}}{\partial \bar{X}_{j}(\bar{t})}=0, j=1,2, \ldots, 5 n^{2}$ is a system of equations for the static problem [12].

The system must be solved under the initial conditions at $\bar{t}=0$ :

$$
U_{k l}=V_{k l}=W_{k l}=\Psi_{x k l}=\Psi_{y k l}=0, \quad \dot{U}_{k l}=\dot{V}_{k l}=\dot{W}_{k l}=\dot{\Psi}_{x k l}=\dot{\Psi}_{y k l}=0, \quad k, l=\overline{1, n} .
$$

When choosing the approximating functions (9) and (17), the boundary conditions were considered, which are the conditions for shell contour support.

The process of forming the system (19) was programmed using the Maple analytical software package. The resulting ODE system was solved numerically using the Rosenbrock method [14], which is effective in solving rigid systems.

\section{NUMERICAL RESULTS}

\subsection{STATIC PROBLEMS}

Let us analyze a doubly-curved shallow shell (Figure 1), square in plan $\left(A=1, B=1, R_{1}=\right.$ const, $R_{2}=$ const ), with the following characteristics: $h=0.09 \mathrm{~m}, a=18 \mathrm{~m}, b=18 \mathrm{~m}, R_{1}=R_{2}=45.27 \mathrm{~m}$ and simply supported along the contour $\left(U=V=W=M_{x}=\Psi_{y}=0\right.$ and $U=V=W=M_{y}=\Psi_{x}=0$ at edges). Loading: uniformly distributed along the normal to the surface $\left(q=\right.$ const,$\left.P_{x}=P_{y}=0\right)$. Material: steel $\left(E_{1}=E_{2}=2.1 \cdot 10^{5} \mathrm{MPa}, \mu_{12}=\mu_{21}=0.3, G_{12}=G_{13}=G_{23}=0.807 \cdot 10^{5} \mathrm{MPa}\right)$. The calculations were performed for $n=4$.

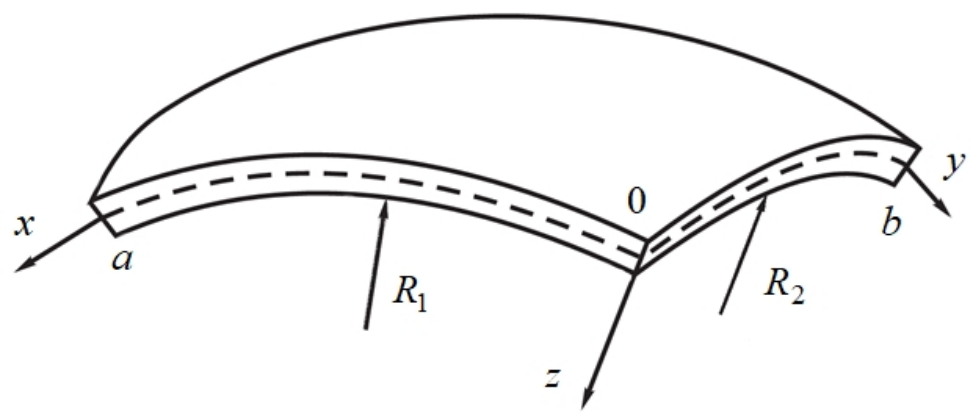

Fig. 1 Schematic representation of the doubly-curved shallow shell

Figure 2 shows the obtained load/deflection diagram. For static problems, the points of maximum on the graphs correspond to the values of the upper critical buckling load. Also, the critical load corresponds to the state of the system when $\operatorname{det}(J)=0$. Here and further, the red curve $W_{c}$ in the diagrams depicts the deflection in the center of a structure 
$\left(x=\left(a_{1}+a\right) / 2, y=b / 2\right)$, and the blue curve $W_{4}$ the deflection in the quadrant of a structure $\left(x=\left(3 a_{1}+a\right) / 4, y=b / 4\right)$ The obtained buckling load value is equal to $q_{c r}=0.6238$.

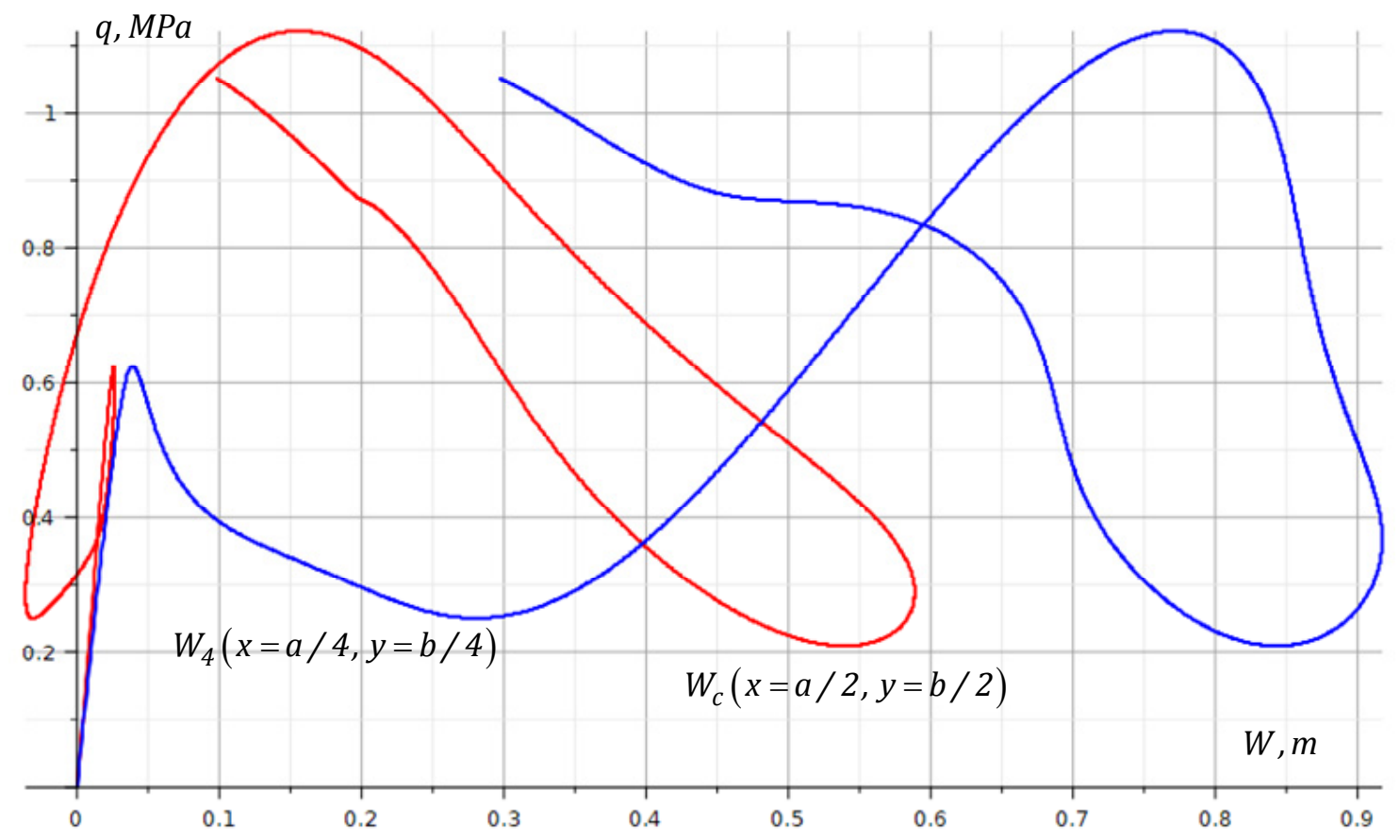

Fig. 2 Calculation results for the doubly-curved shallow shell

\subsection{DYNAMIC PROBLEMS}

Let us analyze a panel of a truncated conical shell (Figure 3) $\left(A=1, B=x \sin (\theta), R_{1}=\right.$ infinity, $R_{2}=x \tan (\theta), \theta$ denotes taper angle) with the following characteristics: $h=0.08 \mathrm{~m}, a 1=16 \mathrm{~m}, a$ $=32 \mathrm{~m}, b=2.574 \mathrm{rad}$ and simply supported along the contour $\left(U=V=W=M_{x}=\Psi_{y}=0\right.$ and $U=$ $V=W=M_{y}=\Psi_{x}=0$ at edges). Loading: uniformly distributed along the normal to the surface ( $q=A_{1} t, P_{x}=P_{y}=0$ ). Loading rate: in the range from $A_{1}=1$ to $A_{1}=1000 \mathrm{MPa} / \mathrm{s}$. Material: E-Glass Epoxy $\left(E_{1}=0.607 \cdot 10^{5} \mathrm{MPa}, \mu_{12}=0.23, E_{2}=0.248 \cdot 10^{5} \mathrm{MPa}, G_{12}=0.12 \cdot 10^{5} \mathrm{MPa}, \rho=1800\right.$ $\mathrm{kg} / \mathrm{m}^{3}$ ). The calculations were performed for $n=4$.

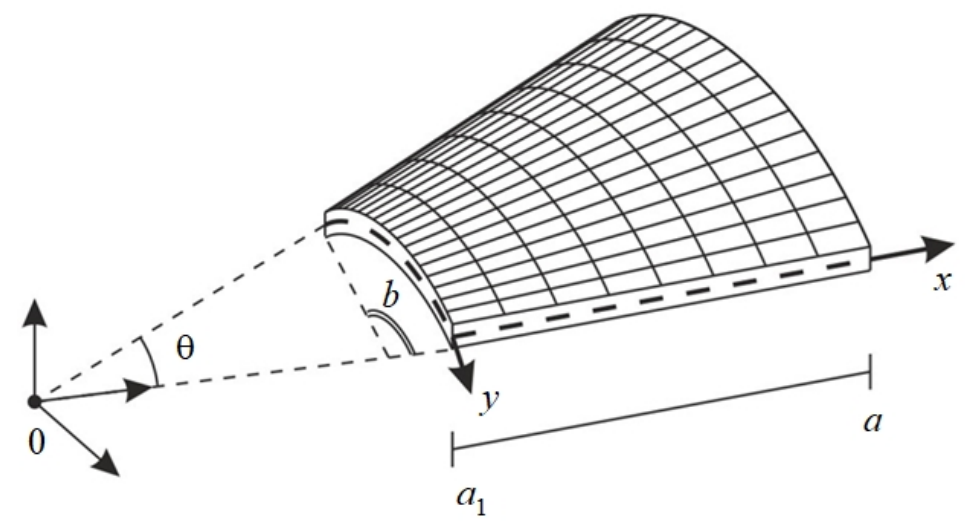

Fig. 3 Schematic representation of a panel of the truncated conical shell 
The process of buckling a shell under dynamic loading differs from the process of buckling in statics. Although criteria for the buckling are based on the Lyapunov (with a small change in the input data, a significant change in the solution of a problem occurs), a different criterion for the buckling is used in dynamics. The load at the inflection point of the "load-deflection" curve is a criterion for the stability loss under dynamic loading (proposed by Volmir).

A comparison of "load-deflection" curves for the examined shell made of E-Glass Epoxy, depending on the rate of loading (the upper index in the diagram notations) is shown in Figure 4. The obtained buckling load value at $A_{1}=1 \mathrm{MPa} / \mathrm{s}$ is equal to $q_{c r}=0.6755 \mathrm{MPa}$, at $A_{1}=10 \mathrm{MPa} / \mathrm{s}$ is equal to $q_{c r}=1.3305 \mathrm{MPa}$ and at $A_{1}=100 \mathrm{MPa} / \mathrm{s}$ is equal to $q_{c r}=4.8099 \mathrm{MPa}$. For a loading rate of $A_{1}=1000 \mathrm{MPa} / \mathrm{s}$, buckling occurs at a load of $q_{c r}=22.1055 \mathrm{MPa}$ (due to the large scale, not shown in the figure).

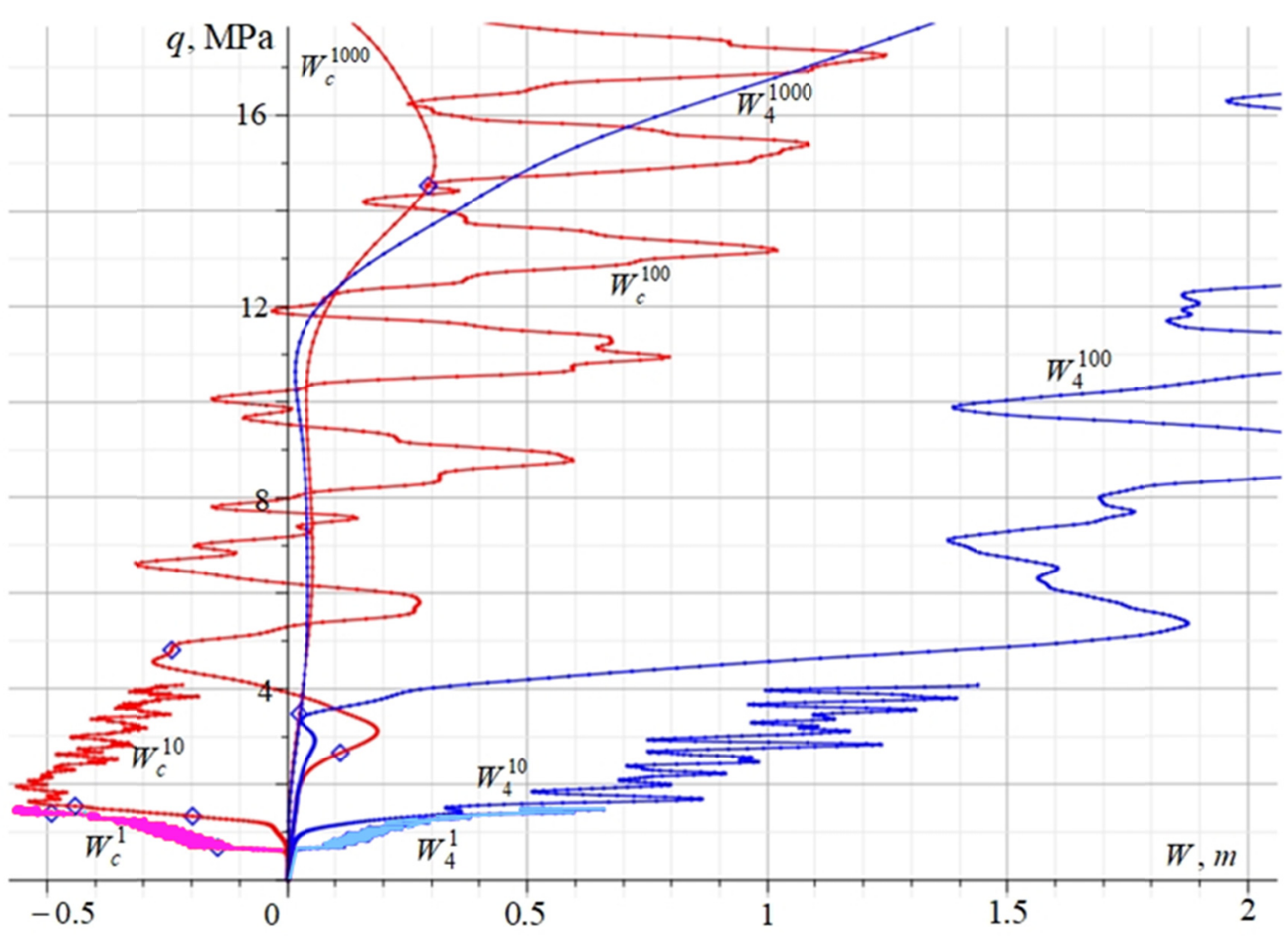

Fig. 4 Calculation results for a panel of the truncated conical shell at different loading rates

\section{CONCLUSION}

In this paper, we presented the example of performing a computational experiment in studying shell structures under static and dynamic loading. The experiment was based on the geometrically nonlinear mathematical model. During the study, we used effective numerical methods that allowed us to get the desired result.

The calculation algorithm based on the Ritz method, the best parameter continuation method, and the Euler method makes it possible to obtain buckling load values and data on structure deformation, even in the post-buckling state. 
The calculation algorithm based on the Kantorovich and Rosenbrock methods makes it possible to obtain data on structure deformation under dynamic loading and solve a rigid ODE system in the post-buckling state.

Therefore, a computational experiment with the implementation of the former approaches allows researchers to perform a comprehensive analysis of structural elements.

\section{REFERENCES}

[1] H.-G. Fan, Z.-P. Chen, W.-Z. Feng, F. Zhou, G.-W. Cao, Dynamic Buckling of Cylindrical Shells with Arbitrary Axisymmetric Thickness Variation under Time Dependent External Pressure, International Journal of Structural Stability and Dynamics, 2015, Vol. 15, No. 3 , P. 1450053. https://doi.org/10.1142/S0219455414500539

[2] S.S. Gavryushin, A.S. Nikolaeva, Method of change of the subspace of control parameters and its application to problems of synthesis of nonlinearly deformable axisymmetric thin-walled structures, Mechanics of Solids, 2016, Vol. 51, No. 3, P. 339-348.

https://doi.org/10.3103/S0025654416030110

[3] Ya.M. Grigorenko, Yu.A. Avramenko, Refined Stress Analysis of Orthotropic Toroidal Shells, International Applied Mechanics, 2013, Vol. 49, No. 4, P. 461-474.

https://doi.org/10.1007/s10778-013-0580-z

[4] V.V. Karpov, Models of the shells having ribs, reinforcement plates and cutouts, International Journal of Solids and Structures, 2018, Vol. 146, P. 117-135.

https://doi.org/10.1016/j.ijsolstr.2018.03.024

[5] V. Karpov, Variational method for derivation of equations of mixed type for shells of a general type, Architecture and Engineering, 2016, Vol. 1, No. 2, P. 43-48.

https://doi.org/10.23968/2500-0055-2016-1-2-43-48

[6] V.V. Karpov, A.A. Semenov, Dimensionless models of deformation of stiffened shell structures, PNRPU Mechanics Bulletin, 2018, No. 1-2, P. 37-49.

https://doi.org/10.15593/perm.mech/eng.2018.1.05

[7] Y. Kumar, The Rayleigh-Ritz method for linear dynamic, static and buckling behavior of beams, shells and plates: A literature review, Journal of Vibration and Control, 2017, P. 107754631769472. https://doi.org/10.1177/1077546317694724

[8] E.B. Kuznetsov, Continuation of solutions in multiparameter approximation of curves and surfaces, Computational Mathematics and Mathematical Physics, 2012, Vol. 52, No. 8 , P. 1149-1162. https://doi.org/10.1134/S0965542512080076

[9] K.M. Liew, Y.Y. Lee, T.Y. Ng, X. Zhao, Dynamic stability analysis of composite laminated cylindrical panels via the mesh-free kp-Ritz method, International Journal of Mechanical Sciences, 2007, Vol. 49, No. 10, P. 1156-1165.

https://doi.org/10.1016/j.ijmecsci.2007.02.005

[10] V.A. Maksimyuk, E.A. Storozhuk, I.S. Chernyshenko, Variational finite-difference methods in linear and nonlinear problems of the deformation of metallic and composite shells (review), International Applied Mechanics, 2012, Vol. 48, No. 6, P. 613-687.

https://doi.org/10.1007/s10778-012-0544-8 
[11] A. Semenov, Mathematical model of deformation of orthotropic shell structures under dynamic loading with transverse shears, Computers \& Structures, 2019, Vol. 221, P. 6573. https://doi.org/10.1016/i.compstruc.2019.05.017

[12] A.A. Semenov, Strength and stability of geometrically nonlinear orthotropic shell structures, Thin-Walled Structures, 2016, Vol. 106, P. 428-436.

https://doi.org/10.1016/j.tws.2016.05.018

[13] F. Shadmehri, S.V. Hoa, M. Hojjati, Buckling of conical composite shells, Composite Structures, 2012, Vol. 94, No. 2, P. 787-792.

https://doi.org/10.1016/j.compstruct.2011.09.016

[14] L.F. Shampine, R.M. Corless, Initial value problems for ODEs in problem solving environments, Journal of Computational and Applied Mathematics, 2000, Vol. 125, No. 12, P. 31-40. https://doi.org/10.1016/S0377-0427(00)00456-8

[15] S.N. Sklepus, Numerical-Analytical Method of Studying Creep and Sustained Strength Characteristics of a Multilayer Shell, Strength of Materials, 2017, Vol. 49, No. 2, P. 313319. https://doi.org/10.1007/s11223-017-9871-7

[16] Z. Su, G. Jin, S. Shi, T. Ye, A unified accurate solution for vibration analysis of arbitrary functionally graded spherical shell segments with general end restraints, Composite Structures, 2014, Vol. 111, P. 271-284.

https://doi.org/10.1016/j.compstruct.2014.01.006

[17] S.N. Timergaliev, A.N. Uglov, L.S. Kharasova, Solvability of geometrically nonlinear boundaryvalue problems for shallow shells of Timoshenko type with pivotally supported edges, Russian Mathematics, 2015, Vol. 59, No. 5, P. 41-51.

https://doi.org/10.3103/S1066369X15050060

[18] S. Trushin, Numerical algorithm for solving of nonlinear problems of structural mechanics based on the continuation method in combination with the dynamic relaxation method, MATEC Web of Conferences / ed. Andreev V, 2016, Vol. 86, P. 01006.

https://doi.org/10.1051/matecconf/20168601006

[19] L.N. Yshii, R.C. Santana, F.A.C. Monteiro, E. Lucena Neto, Buckling of Cylindrical Panels by a Ritz Scheme, 2017. https://doi.org/10.20906/CPS/CILAMCE2017-0613

[20] E.I. Grigolyuk, E.A. Lopanitsyn, Influence of axisymmetric initial irregularities of a spherical shell on its critical load, Izvestija Moskovskogo gosudarstvennogo tehnicheskogo universiteta MAMI, 2008, No. 1, P. 233-246. (in Russian)

[21] E.I. Grigoljuk, V.I. Shalashili, Metod prodolzhenija reshenija po parametru v nelinejnyh zadachah mehaniki tverdogo deformiruemogo tela (Method for the continuation of a solution with respect to a parameter in nonlinear problems in the mechanics of a solid deformable body), Moscow: Nauka, 1988, 232 p. (in Russian)

[22] V.I. Shalashilin, E.B. Kuznecov, Metod prodolzhenija reshenija po parametru i nailuchshaja parametrizacija $\mathrm{v}$ prikladnoj matematike i mehanike (Method for the continuation of a solution by a parameter and the best parameterization in applied mathematics and mechanics), Moscow: Jeditorial URSS, 1999, 224 p. (in Russian) 